\title{
Corrigendum: Evolutionary instability of zero-determinant strategies demonstrates that winning is not everything
}

\author{
Christoph Adami \& Arend Hintze
}

Nature Communications 4:2193 doi: 10.1038/ncomms3193 (2013); Published 1 Aug 2013; Updated 16 Jun 2014

It has been drawn to our attention that the instability found here for zero determinant (ZD) strategies only applies to the set of coercive strategies we considered, which is a subset of all possible ZD strategies. In the Article, we pointed out that the set of ZD strategies investigated by Press and Dyson ${ }^{1}$ was not stable under Darwinian evolution. We speculated that the instability we noted would hold for the extended set also; however, as discussed by Stewart and Plotkin, 2013 (see also Hilbe et al., 2013), this speculation was unwarranted because the ZD strategies not studied by Press and Dyson are generous and therefore stable in an evolutionary sense.

Stewart, A. J. \& Plotkin, J. B. From extortion to generosity, evolution in the Iterated Prisoner's Dilemma. Proc. Natl Acad. Sci. USA 110, 15348-15353 (2013). Hilbe, C., Nowak, M. A. \& Traulsen, A. Adaptive dynamics of extortion and compliance. PLoS ONE 8, e77886 (2013). 